Nervenarzt $2007 \cdot 78: 62-73$

DOI 10.1007/s00115-005-1974-x

Online publiziert: 3. August 2005

๑) Springer Medizin Verlag 2005

\author{
D. Müller $\cdot$ V. Roder $\cdot H$. Brenner \\ Universitätsklinik für Sozial- und Gemeindepsychiatrie, \\ Universitäre Psychiatrische Dienste, Bern
}

\title{
Effektivität des Integrierten Psychologischen Therapieprogramms für schizophren Erkrankte
}

\section{Eine Metaanalyse über 28 unabhängige Studien}

In der psychiatrischen Versorgung basiert heute die Behandlung schizophren Erkrankter auf eine Therapietrias. Diese stützt sich neben (unspezifischer) soziotherapeutischer Rehabilitation weitgehend auf die beiden Bereiche Psychopharmakotherapie und Psychotherapie. In diesen Bereichen wie auch in den allgemeinen Rahmenbedingungen zeichneten sich während des letzten Jahrzehnts wesentliche Entwicklungen ab:

- In der psychiatrischen Versorgung setzte ein Paradigmawechsel von der vorwiegend stationären hin zu vermehrter ambulanter und gemeindeintegrierter Behandlung ein $[1,2,8]$.

- Die Pharmakotherapie erreichte mit der Entwicklung so genannter atypischer Neuroleptika große Fortschritte $[18,25,44]$.

- m Rahmen der psychotherapeutischen Behandlung konnten kognitiv-verhaltenstherapeutische Interventionen ihre Überlegenheit im Vergleich mit anderen Methoden nachweisen $[40,45,76]$.

Kognitiv-verhaltenstherapeutische Ansätze lassen sich entsprechend ihren Zielbereichen in vier Hauptrichtungen gliedern $[50,51,55]$ :

1. soziale Fertigkeits- und Problemlösetrainings (SST),
2. kognitive Remediation,

3. kognitiv-behaviorale Ansätze zur Reduzierung persistierender Positivsymptomatik (CBT),

4. psychoedukative und familientherapeutische Ansätze.

Verschiedene Metaanalysen weisen die Effektivität dieser Ansätze nach [23, 35, 39, $45,51,52]$. Das Integrierte Psychologische Therapieprogramm (IPT) $[9,56]$ wird in diesen Metaanalysen schwerpunktmäßig den ersten beiden Hauptrichtungen zugeordnet.

\section{Das Integrierte Psychologische Therapieprogramm}

Das IPT stellt einen der ersten systematischen, empirisch fundierten und manualisierten Behandlungsansätze für schizophren erkrankte Patienten dar. Die Übersetzung des Therapiemanuals in 10 Sprachen und die bereits 5 . Auflage in deutscher Sprache [55] deuten auf eine breite und anhaltende Akzeptanz dieses kognitiv-behavioralen Gruppentherapieprogramms hin. Das IPT besteht aus 5 Unterprogrammen (• Abb. 1).

Die mehrstufigen Unterprogramme (UP) werden sequenziell durchgearbeitet. Die ersten beiden UP bearbeiten vor allem grundlegende Defizite in den Berei- chen Neurokognitionen und soziale Kognitionen. Die Bearbeitung neurokognitiver Defizite unterscheidet sich dabei vom herkömmlichen, wiederholten Training so genannter „kalter“ Kognitionen, da entsprechende Übungen im Kontext der Gruppeninteraktionen durchgeführt werden. Die letzten 3 UP fokussieren das ressourcenbezogene (Wieder-)Erlangen sozialer Kompetenz.

Das therapeutische Konzept des IPT wurde in den Bereichen der sozialen Kognitionen und des Sozial- und Problemlöseverhaltens durch zwei Weiterentwicklungen ergänzt: dem Therapieansatz zur Bewältigung von maladaptiven Emoti-

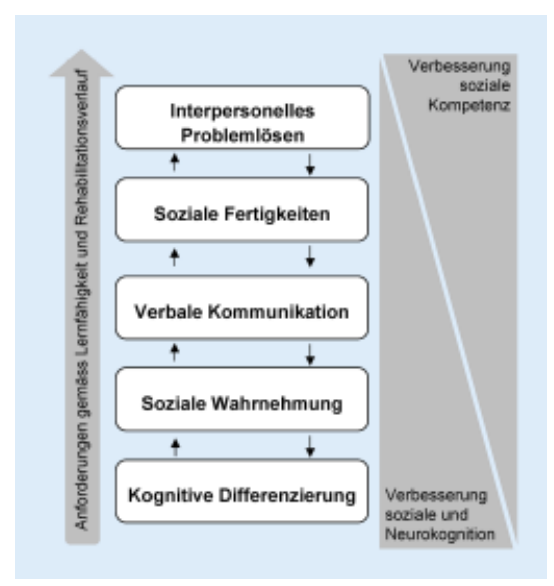

Abb. $1 \Delta$ Integriertes Psychologisches Therapieprogramm 
onen (TBE) [31] und den spezifischen Therapieprogrammen in den Bereichen Arbeit, Wohnen und Freizeit (WAF) [54, 58].

\section{Methode}

Während der letzten 24 Jahre führten verschiedene Arbeitsgruppen aus der Schweiz, den USA, Kanada, Japan, Deutschland, den Niederlanden, Italien und Spanien 28 unabhängige Evaluationsstudien zum gesamten IPT oder zu Kombinationen einzelner UP durch. Dabei wurden insgesamt 1329 schizophren erkrankte Patienten einbezogen (• Tab. 1). Fünf Studien verwendeten die Diagnosekriterien nach DSM-III, je 4 Studien nach DSM-III-R bzw. DSM-IV, 10 Studien nach ICD-9 und 3 Studien nach ICD-10. Eine Studie setzte ein alternatives Diagnosesystem (Research Diagnostic Criteria, RDC [66]) und eine Studie machte keine Angaben.

In 2 Studien wurde das IPT sowohl mit einer Standardbehandlung basierend auf Pharmako- und Soziotherapie (Leerkontrollgruppe) als auch mit Plazebo-Attention-Bedingungen (unspezifische Gruppenaktivitäten mit identischem Umfang und Intensität wie die IPT-Gruppen) verglichen, in 11 Studien mit einer Standardbehandlung, in 8 Studien mit PlazeboAttention-Bedingungen und in 2 Studien stellte das IPT die Kontrollgruppe für einen anderen Therapieansatz (Training emotionaler Intelligenz, TEI [72]; soziale Fertigkeiten zum Wohn-, Arbeits- und Freizeitbereich WAF [59]) dar. Fünf weitere Studien verzichteten auf eine Kontrollgruppe (Feldstudien) oder gingen Fragestellungen zur differenziellen Indikation nach.

Das IPT wurde mit Patienten mit unterschiedlichem Rehabilitationsstatus unter verschiedenen Versorgungsbedingungen angewendet: symptomstabilisierte Patienten, in Anlehnung an die ICD10-Zusatzkodierungen definiert als Patienten mit kontinuierlichen Symptomen (F20.xo) oder mit Residualsymptomen zwischen den Episoden (stabiler Residualzustand) (F2O.X2) bei vorliegender therapeutischer Indikationsstellung, oder Patienten in einer postakuten Remissionsphase nach einer Exazerbation aus dem stati-

Nervenarzt 2007 ·78:62-73 DOI 10.1007/ s00115-005-1974-x

(c) Springer Medizin Verlag 2005

D. Müller $\cdot$ V. Roder $\cdot H$. Brenner

Effektivität des Integrierten Psychologischen

Therapieprogramms für schizophren Erkrankte. Eine Metaanalyse über $\mathbf{2 8}$ unabhängige Studien

\section{Zusammenfassung}

Während der letzten 24 Jahre führten Arbeitsgruppen aus 8 Ländern 28 unabhängige Evaluationsstudien zum Integrierten Psychologischen Therapieprogramm (IPT) mit insgesamt 1329 schizophren Erkrankten durch. Zur Überprüfung der Wirksamkeit des IPT unter verschiedenen Versorgungsbedingungen wurden die inferenzstatistischen Ergebnisse sämtlicher Studien in Effektstärken transformiert. Die IPT-Gruppen zeigen einen signifikant höheren mittleren Therapieeffekt gegenüber den Kontrollgruppen (Plazebo-Attention-Bedingungen; Standardbehandlung). Die Überlegenheit der IPT-Gruppen nimmt im Verlauf einer durchschnittlichen Katamnesedauer von 8,1 Monaten weiter zu. Vergleichbar hohe Effekte lassen sich für die IPTGruppen sowohl in verschiedenen Funktionsbereichen (kognitive Fähigkeiten, Sozialver-

\section{Effectiveness of Integrated Psychological Therapy for schizophrenia patients. A meta-analysis including 28 independent studies}

\section{Summary}

Over the past 24 years, research groups in eight different countries have conducted 28 independent evaluation studies on Integrated Psychological Therapy (IPT) including 1,329 schizophrenia patients. The present study examines the effectiveness of IPT under different treatment conditions by means of a meta-analytic review. The most salient results indicate favourable mean effect sizes for IPT in comparison to control groups (placebo-attention conditions, standard care). Moreover, the superiority of IPT continues to increase during an average catamnestic phase of 8.1 months. The method obtains similarly favourable effects in different functional areas (neurocognition, social behaviour, psychopathology) and different assessment formats (expert ratings, self-reports, psychological tests). The halten, Psychopathologie) als auch mit unterschiedlichen Erhebungsformaten (psychologische Tests, standardisierte Selbstund Fremdratings) nachweisen. Die Ergebnisse belegen weiter, dass Patienten unabhängig von Krankheits-, Rehabilitationsstatus und Therapiesetting vom IPT profitieren. Alle Ergebnisse sind mittels einer Selektion von qualitativ hochstehenden Studien validiert. Zusammenfassend unterstützen damit die Ergebnisse die Evidenz des IPT als einen „empirisch validierten Treatmentansatz" im Sinne der APA-Richtlinien.

Schlüsselwörter

Schizophrenie · Kognitiv-verhaltenstherapeutische Psychotherapie - Versorgungsbedingungen $\cdot$ Rehabilitation $\cdot$ Metaanalyse comparison of different settings of IPT and control groups shows the superiority of IPT in any given therapy or site condition. The analysis of subsamples of inpatients, outpatients, and patients in varying rehabilitation phases reveals similarly favourable effects. Comparing only high-quality studies yields comparable results. In summary, the present metaanalysis corroborates evidence that IPT is an 'empirically validated treatment' according to American Psychiatric Association guidelines.

\section{Keywords}

Schizophrenia $\cdot$ Cognitive-behavioural psychotherapy - Treatment conditions . Rehabilitation · Meta-analysis 
onären und ambulanten Bereich von universitären und nichtuniversitären Institutionen. Die Qualität der Studien ist hinsichtlich Stichprobengröße und Design heterogen: 23 Studien $(82,1 \%)$ weisen ein kontrolliertes Design auf, wovon 14 Studien $(60,8 \%)$ eine randomisierte Patientenzuweisung vornahmen. Bei 22 Studien $(78,6 \%)$ sind auch auf Fremdbeurteilung gestützte Messmittel eingesetzt worden und bei 6 dieser Studien (27,3\%) entsprechen diese Blindratings. Therapiesetting und Design der Studien unterscheiden sich über den Publikationszeitraum von 24 Jahren: je älter die Studien desto höher die Therapiefrequenz (K-Studien $=28$, Spearmans Rangkorrelation, zweiseitig: $r=-0,56, p<0,01)$ und desto geringer die Stichprobengröße $(K=28, r=0,41$, $p=0,03)$. Entsprechend unterscheidet sich die Therapiefrequenz auch unter Berücksichtigung der chronologischen Abfolge der DSM- und ICD-Ausgaben: Studien mit einer Diagnosestellung nach DSMIII oder ICD-9 weisen eine signifikant geringere Therapiefrequenz auf, als Studien nach DSM-III-R, DSM-IV oder ICD10 (ANOVA: $F=5,14 ; \mathrm{df}=4,21 ; p<0,01$, post hoc: LSD: $p<0,03)$.

Zur systematischen Erfassung und vollständigen Zusammenfassung vorliegender Forschungsergebnisse zum IPT und zur quantitativen Analyse der Befundvariabilität werden zunächst sämtliche vorliegende IPT-Studien in die Analyse integriert. Damit wird die Wirksamkeit des IPT unter verschiedenen realen klinischen Versorgungsbedingungen („effectiveness") überprüft und der Forderung nach Einbezug von nichtuniversitären Institutionen in die wissenschaftliche Evaluation sowie dem Nachweis von Effekten in der klinischen Realität mittels Feldstudien [69] entsprochen. Die Vergleichbarkeit der 28 IPT-Studien hängt entsprechend von deren designund messmittelbedingten methodischen Qualität und dem Stichprobenfehler der eingeschlossenen Studienpopulationen ab. Dabei unterscheidet sich die vorliegende Arbeit gegenüber anderen Metaanalsen zu psychotherapeutischen Interventionen, da die Experimentalgruppen aller Primärstudien ausschließlich konzeptuell identische, hoch standardisierte und manualisierte psychotherapeutische
Intervention des IPT einsetzten. Eine einheitliche Implementierung des IPT wurde durch den persönlichen Kontakt und/ oder Therapeutenschulungen der Studienverantwortlichen mit unserer Arbeitsgruppe zusätzlich gewährleistet. Die qualitative Heterogenität der Studien hinsichtlich der Patientenmerkmale, Design, Setting und Messmittel wird anhand entsprechender Kategorisierung der Studien kontrolliert. Zusätzliche unsystematische und nicht dokumentierte Einflussbzw. Störvariablen (z. B. zusätzliche pharmako-, psycho- und soziotherapeutische Behandlung) werden durch die systematische Varianzaufklärung und die Überprüfung der Effektkonsistenz der Metaanalyse indirekt berücksichtigt.

Zusammenfassend sind folgende Fragestellungen von Interesse:

- der globale Therapieeffekt (mittlerer

Effekt aller erhobenen Outcomevari-

ablen) der Therapie- und Katamnesephase,

- differenzielle Effekte in den 3 zentralen Funktionsbereichen schizophren

Erkrankter (kognitiver Bereich, Sozialverhalten und Psychopathologie),

- Moderatoren/Einflussbedingungen

(Patienten- und Rahmenbedingungen),

- Outcomeprädiktoren.

In einem zweiten Schritt wird zusätzlich der mögliche Einfluss der methodischen Qualität auf die Ergebnisse aller 28 Studien überprüft. Dabei dient die Überprüfung der internen Validität der einzelnen Studien als Selektionskriterium. Es werden ausschließlich Studien mit einer Dokumentation der Diagnostik und randomisierter Patientenzuweisung (Randomized Controlled Trials, RCT) und Studien mit zusätzlich kontrollierter Erfassung der Medikation und blinde oder keine Fremdbeurteilungen (High Quality Studies HQS) in die Validierung eingeschlossen.

\section{Datenanalyse}

Um alle IPT-Studien in die Analyse einzubeziehen, werden zunächst Effektstärken (ES) innerhalb der Vergleichsgruppen nach Smith u. Glass [64] berechnet: Die Mittelwertsdifferenz der Prä- und Postwerte (oder Follow-up-Werte) jeder Vergleichsgruppe wird ins Verhältnis zur Standardabweichung der gepoolten Baseline der Experimental- und Kontrollgruppe gesetzt [ $\left(M_{\text {post }}-M_{\text {prä }}\right) /$ $S D_{\text {prägepoolt }}$. $E S=0,5$ entspricht somit einer durchschnittlichen Verbesserung der Gruppe von einer halben Standardabweichung der Gesamtpopulation zu Therapiebeginn. Da verschiedene Studien auf größere Effekte von Patienten unter Plazebo-Attention-Bedingungen hinweisen als bei Patienten mit Standardbehandlung [52, 78], werden die beiden Kontrollbedingungen gesondert betrachtet. Ergänzend werden ES zwischen den Gruppen ermittelt (Cohens $d$ ) [17]: Die statistischen Kennwerte werden anhand folgender Formel in Cohens $d$ transformiert: $d=2(F / d f)^{1 / 2}$. ES und $d$ können in klein $(0,2)$, mittel $(0,5)$ und groß $(0,8)$ kategorisiert werden [17]. Die Kontrolle möglicher Einfüsse durch ungleiche Stichprobengrößen und Standardfehler erfolgt zunächst auf der Grundlage eines kontinuierlichen Modells fester Effekte („fixed effects model“): Die ES der einzelnen Studien werden anhand ihrer inversen konditionalen Varianzen gewichtet $\left(E S_{\mathrm{w}}=w \times E S\right.$; $d_{\mathrm{w}}=w \times d$ : Gewichtungsfaktor $w=1 / s_{\mathrm{i}}{ }^{2} / \sum 1 /$ $s_{\mathrm{i}}{ }^{2}$, wobei $1 / s_{\mathrm{i}}{ }^{2}=$ Varianzkehrtwert einer Primärstudie und $\sum 1 / s_{\mathrm{i}}{ }^{2}=$ Summe der Varianzkehrtwerte aller integrierten Studien darstellen) [63]. Die Signifikanzprüfung von $E S_{\mathrm{w}}$ und $d_{\mathrm{w}}$ erfolgt über die Berechnung des Konfidenzintervalls (CI) bzw. eine Z-Standardisierung der Effekte ( $p<0,05$, wenn $95 \% \mathrm{CI}$ von $E S_{\mathrm{w}}$ oder $d_{\mathrm{w}}$ nicht o einschließt). Die Überprüfung der Homogenität der integrierten gewichteten Effekte $\left(E S_{\mathrm{w}}, d_{\mathrm{w}}\right)$ erfolgt über die Berechnung von Hedges' $Q_{W}$ (= Summe der gewichteten quadrierten Abweichungen der einzelnen Studieneffekte vom mittleren Integrationseffekt). Die Signifikanzprüfung von $Q_{W}$ basiert auf einer $\chi^{2}$-Verteilung mit $\mathrm{DF}=p-1$ Freiheitsgraden. Gruppenunterschiede (z. B. zwischen Experimental- und verschiedenen Kontrollbedingungen) werden mittels der Berechnung von Hedges' $Q_{B}$ (nach der Formel: $\left.Q_{B}=Q_{\text {Total }}-Q_{W}\right)$ überprüft [26]. Signifikanz- und Homogenitätsstatistik erfolgen analog zu jenen von $Q_{\mathrm{w}}$.

Die mit dem Modell fester Effekte generierten signifikanten Ergebnisse werden 


\begin{tabular}{|c|c|c|c|c|c|c|}
\hline Autoren & Land & Intervention & $n$ & Setting & Krankheitsstatus & Zentrum \\
\hline Brenner et al. $[7,10]$ & D & $\mathrm{IPT}^{\mathrm{a}}$ & 43 & Stationär & Symptomstabilisiert ${ }^{\text {h }}$ & Universitär \\
\hline Brenner et al. [11] & D & UP4 bzw. UP2 $2^{b}$ & 28 & Stationär & Symptomstabilisiert & Universitär \\
\hline Stramke und Hodel [66] & $\mathrm{CH}$ & UP2 & 18 & Stationär & Symptomstabilisiert & Universitär \\
\hline Bender et al. [3] & D & UP1+2 & 28 & Stationär & Symptomstabilisiert & Nichtuniversitär \\
\hline Brenner et al. [7] & D & IPT & 18 & Ambulant & Symptomstabilisiert & Nichtuniversitär \\
\hline Hermanutz und Gestrich [29] & D & IPT & 64 & Stationär & Postakut & Nichtuniversitär \\
\hline Kraemer et al. [37] & $\mathrm{D}$ & $U P 1+2+K B^{c}$ & 30 & Stationär & Symptomstabilisiert & Mix \\
\hline Roder et al. [57] & $\mathrm{CH}$ & IPT & 17 & Stationär & Symptomstabilisiert & Nichtuniversitär \\
\hline Funke et al. [20] & $\mathrm{D}$ & UP1+2 & 24 & Stationär & Symptomstabilisiert & Nichtuniversitär \\
\hline Heim et al. [27] & $\mathrm{D}$ & UP1-3 & 65 & Stationär & Symptomstabilisiert & Nichtuniversitär \\
\hline Peter et al. $[48,49]$ & D & UP1-3 & 83 & Stationär & Postakut & Universitär \\
\hline Hodel [30] & $\mathrm{CH}$ & IPT & 21 & Stationär & Symptomstabilisiert & Universitär \\
\hline Kraemer et al. [38] & $\mathrm{D}$ & UP1+2 vs UP4 & 43 & Stationär & Symptomstabilisiert & Universitär \\
\hline Olbrich und Mussgay [46] & $\mathrm{D}$ & UP1 & 30 & Stationär & Postakut & Universitär \\
\hline Roder et al. [53] & $\mathrm{CH}$ & UP1 & 18 & Stationär & Symptomstabilisiert & Nichtuniversitär \\
\hline $\begin{array}{l}\text { Schüttler et al. [62], } \\
\text { Blumenthal et al. [5] }\end{array}$ & $\mathrm{D}$ & UP1-4 & 95 & Stationär & Postakut & Nichtuniversitär \\
\hline Hubmann et al. [33] & $\mathrm{D}$ & UP4+Token ${ }^{d}$ & 21 & Stationär & Symptomstabilisiert & Nichtuniversitär \\
\hline Gaag van [21] & NL & UP1+2 mod. & 42 & Stationär & Symptomstabilisiert & Nichtuniversitär \\
\hline Takai et al. [68] & J & IPT & 34 & Stationär & Symptomstabilisiert & Mix \\
\hline Theilemann [70] & $\mathrm{D}$ & IPT & 45 & Stationär & Postakut & Nichtuniversitär \\
\hline Vallina et al. [72] & $\mathrm{E}$ & UP2-4+PE & 35 & Ambulant & Symptomstabilisiert & Nichtuniversitär \\
\hline Spaulding et al. [65] & USA & UP1-3+SST & 91 & Stationär & Symptomstabilisiert & Universitär \\
\hline Roder et al. [59] & $\mathrm{CH}$ & UP4 & 143 & Mix & Symptomstabilisiert & Mix \\
\hline Vauth et al. [73] & $\mathrm{CH}$ & UP4+5 & 57 & Stationär & Postakut & Universitär \\
\hline Vita et al. [75] & I & IPT & 86 & Ambulant & Symptomstabilisiert & Nichtuniversitär \\
\hline Briand et al. [12] & CDN & IPT+TBE & 90 & Mix & Mix & Mix \\
\hline Penadés et al. [47] & $E$ & UP1+2 & 37 & Ambulant & Symptomstabilisiert & Universitär \\
\hline García et al. [22] & $E$ & UP2 & 23 & Ambulant & Symptomstabilisiert & Nichtuniversitär \\
\hline
\end{tabular}

zusätzlich über die Berechnung einer Varianzkomponente $\left(\Gamma^{2}\right)$ mittels eines Modells zufallsvariabler Effekte (,random effects model") überprüft [63]. Mit diesem Modell werden auch unsystematische, zufallsbedingte Varianzen der Primärstudien und somit deren Stichprobenfehler berücksichtigt, was der Annahme eines konstanten Populationseffektes unter dem Modell fester Effekte widerspricht. Die Varianzkomponente $\left(\Gamma^{2}\right)$ wird nach der Formel $\Gamma^{2}=s^{2}$ ungewichtet $-\sum s_{\mathrm{w}}{ }^{2} / K_{\text {Studien }}$ geschätzt. $\mathrm{Da} \Gamma^{2}$ zur konditionalen Varianz addiert wird, werden die Hypothesenprüfungen in der Regel konservativer und die Vertrauensintervalle breiter. Fällt jedoch die Gesamtvarianz ungewichteter Effekte ( $s^{2}$ ungewichtet) geringer als die durchschnittliche unsystematische Varianz $\left(\sum s_{\mathrm{w}}{ }^{2} / K_{\text {Studien }}\right)$ aus, wird die Varianzkomponente negativ und somit gleich Null gesetzt. Damit reduziert sich das Modell zufallsvariabler Effekte auf jenes fester Effekte mit entsprechend identischen Effekten.

Zur Kontrolle möglicher Publikationsbias wird weiter die Anzahl unpublizierter Studien ohne Effekte berechnet, die benötigt würden, um allfällige positive Ergebnisse zu negieren („faile safe") [6o]. Zur Überprüfung von Zusammenhangs- und Prädiktorhypothesen werden nichtparametrische Spearman-Rangkorrelationen berechnet. Aufgrund der geringen statistischen Power wird auf weitere inferenzstatistische Analysen verzichtet.

\section{Ergebnisse}

Die Patientencharakteristika der $1329 \mathrm{~Pa}-$ tienten aus 28 Studien weisen aufgrund des unterschiedlichen Rehabilitationsstatus der Patienten in den einzelnen Studien heterogene Hospitalisations- und Krankheitsdauern auf (- Tab. 2). Alle Studien verweisen auf eine zusätzliche Pharmakotherapie; acht Studien geben die tägliche Dosierung an.

\section{Therapiesetting und Studienabbrecher}

Die Therapiedauer beträgt 17,4 Wochen (95\%-Konfidenzintervall, CI: 11,9-22,9), 47,4 h (CI: 36,6-58,2) bzw. 44,7 Sitzungen (CI: 37,3-57,5). Die Therapieintensität liegt bei 3,2 Sitzungen pro Woche (CI: 2,7-3,7). 14 Studien nennen Beruf und Qualifikation der Therapeuten. In 92,8\% dieser Studien sind vorwiegend kognitiv-behavioral ausgebildete Psychologen und an 35,7\% ebenfalls im IPT ausgebildete Psychiater als Haupttherapeuten beteiligt. 14 Studien (50\%) nennen die Drop-out-Raten wäh- 
Tab. 2 Patientencharakteristika ( $K=28$ Studien)

\begin{tabular}{|lll|}
\hline & $M$ & $95 \% \mathrm{Cl}^{\mathrm{a}}$ \\
\hline Geschlecht: Männer [\%] & 68,0 & $61,8<\delta<74,2$ \\
\hline Alter [Jahre] & 35,0 & $32,9<\delta<37,0$ \\
\hline IQ & 92,0 & $87,6<\delta<96,4$ \\
\hline Hospitalisationsdauer [Monate] & 77,9 & $40,2<\delta<115,6$ \\
\hline Anzahl Hospitalisationen & 3,9 & $3,6<\delta<4,2$ \\
\hline Krankheitsdauer [Jahre] & 10,2 & $8,1<\delta<12,3$ \\
\hline Tägliche Dosis Psychopharmaka [Chlorpromazinäquivalente] $^{\mathrm{b}}$ & 876,9 & $364,2<\delta<1389,6$ \\
\hline${ }^{a}$ Konfidenzintervall; $^{b} \mathrm{n}=8$ Studien. & & \\
\hline
\end{tabular}

rend der Therapiedauer und 4 dieser Studien machen zusätzliche Angaben für die gesamte Studiendauer (Therapie- und Katamnesephase). Die durchschnittliche Drop-out-Rate beträgt $15,8 \%(\mathrm{SD}=13,4)$ während der Therapiephase und 19,5\% $(\mathrm{SD}=17,7)$ während der gesamten Studiendauer.

\section{Globaler Therapieeffekt und Follow-up innerhalb der Vergleichsgruppen}

Während der Therapiephase zeigen sich mittlere, jedoch signifikant höhere $E S_{\text {w }}$ für die IPT-Gruppen gegenüber den beiden Kontrollbedingungen $\left(Q_{B}=12,59\right.$; $\mathrm{DF}=2, p<0$,o1, einseitig; - Tab. 3). Patienten unter Plazebo-Attention-Bedingungen erreichen kleine $E S_{\text {w }}$, die signifikant von Null abweichen. Diese unterscheiden sich von jenen der Gruppe mit Standardbehandlung marginal $\left(Q_{B}=3,54 ; D F=1, p<0,1\right)$. Die Überlegenheit der IPT-Gruppen bleibt auch während einer durchschnittlichen Katamnesephase von 8,1 Monaten weiter bestehen $\left(Q_{B}=8,29, D F=2, p<0,05\right)$. Die Überprüfung der signifikanten $E S_{\mathrm{w}}$ mit dem Modell zufallsvariabler Effekte erbringt durchwegs unbedeutende Varianzkomponenten $\left(\Gamma^{2} \leq 0\right)$. Fortan wird deshalb auf den Verweis zufallsvariabler Effekte verzichtet.

\section{Differenzielle Effekte in zentralen Funktionsbereichen}

In den drei Bereichen Kognitionen, Sozialverhalten und Psychopathologie (Positiv- und Negativsymptomatik) können signifikante Verbesserungen für die IPTGruppe nachgewiesen werden ( $\bullet$ Tab. 3). Von den beiden Kontrollbedingungen erreicht einzig die Plazebo-Attention-Grup- pe in den Psychopathologievariablen eine signifikante Verbesserung. Ein Vergleich zwischen den Gruppen ergibt eine signifikante Überlegenheit von IPT gegenüber den Kontrollbedingungen im kognitiven Bereich und im Sozialverhalten $\left(Q_{\mathrm{B}}>9,34\right.$; $\mathrm{DF}=2, p<0,01)$, nicht aber in der Psychopathologie $\left(Q_{B}=5,74 ; D F=2, p<0,1\right)$. Werden jedoch die beiden Kontrollbedingungen in diesem Bereich zusammengefasst, weist das IPT signifikant höhere Effekte auf $\left(Q_{\mathrm{B}}=5,19 ; \mathrm{DF}=1, p<0,05\right)$.

\section{Messmittel}

Das IPT erbringt in allen drei Messmittelformaten signifikante Verbesserungen (- Tab. 3), die sich als homogen erweisen $\left(Q_{B}=0,31 ; D F=2, n\right.$. s. $)$. Selbstbeurteilung und Fremdeinschätzung, die ausschließlich Variablen zum Sozialverhalten und zur Psychopathologie beinhalten, erreichen ebenfalls in den Plazebo-AttentionGruppen signifikante Ergebnisse. Selbstund Fremdeinschätzungen stimmen zudem bei den IPT-Patienten hochsignifikant überein (Spearmans Rangkorrelation, zweiseitig: $r=0,74 ; p<0,01 ; K=14)$. Die in den drei Funktionsbereichen am häufigsten eingesetzten Erhebungsinstrumente (Aufmerksamkeits-Belastungs-Test d2 [13], Global Assessment of Functioning Scale (GAF) [61], Brief Psychiatric Rating Scale (BPRS) [16]) erbringen im Mittel $44 \%$ höhere gewichtete Effekte für die IPT-Gruppe als die zu den drei Funktionsbereichen zusammengefassten Variablen. Entsprechend kann in der BPRS im Gegensatz zum zusammengefassten Psychopathologiebereich eine signifikante Überlegenheit des IPT gegenüber den beiden Kontrollbedingungen nachgewiesen wer$\operatorname{den}\left(Q_{B}=6,62 ; D F=2, p<0,05\right)$.
Moderatoren und Einflussbedingungen

\section{Zentren}

Zur Kontrolle und Ausdifferenzierung möglicher Einflüsse durch Institutionen, Behandlungssetting und Krankheitsstatus auf die oben dargestellten Effekte werden die beiden Kontrollbedingungen aufgrund der reduzierten Zellengrößen zusammengefasst (• Tab. 4).

IPT erzielt institutionsunabhängig signifikante Verbesserungen. Die Effekte sind bei IPT- und Kontrollgruppen gröBer in Studien an universitären Zentren als an nichtuniversitären Zentren. In universitären Zentren erreicht zudem auch die Kontrollgruppe signifikante Verbesserungen während der Therapiephase. Die 4 Multicenter-Studien mit überwiegend nichtuniversitärer Partizipation weisen eine größere Varianz, jedoch vergleichbare Effekte zu den nichtuniversitären Zentren auf. Die Unterschiede aufgrund institutioneller Bedingungen sind weder bei den IPT- noch bei der Kontrollgruppen signifikant $\left(Q_{B}<1,24 ; D F=2, n\right.$. s. $)$.

\section{Behandlungssetting}

IPT erzielt bei stationären und ambulanten Patienten signifikante Effekte (• Tab.4). Gegenüber den Kontrollgruppen kann nur bei stationären $\mathrm{Pa}$ tienten eine signifikante Überlegenheit des IPT nachgewiesen werden $\left(Q_{B}=9,33\right.$; $\mathrm{DF}=1, p<0,01)$. Hingegen ist die Überlegenheit des IPT bei ambulanten Patienten marginal $\left(Q_{\mathrm{B}}=3,65 ; \mathrm{DF}=1, p<0,1\right)$. Obwohl stationäre Patienten unter Kontrollbedingungen signifikante Verbesserungen während der Therapiephase aufweisen, unterscheiden sich weder die IPT- noch Kontrollpatienten hinsichtlich des Behandlungssettings $\left(Q_{B}<0,42 ; D F=1, n\right.$. s.). Über die Therapie- und Katamnesephase zeigen stationäre IPT-Patienten höhere gewichtete Effekte (Follow-up $=10$ Monate; $K=4 ; E S_{w}=0,79$; CI: o,43-1,16) als ambulante IPT-Patienten (Follow-up $=7,5 \mathrm{Mo}$ nate; $K=2 ; E S_{\mathrm{w}}=0,44 ; \mathrm{CI}: 0,07-0,80$ ). Während ambulante Patienten ihre während der Therapie erreichten Verbesserungen auch nach deren Abschluss aufrechterhalten können, nehmen die Effekte bei stationären Patienten während der Katamnesephase nochmals signifikant $\mathrm{zu}$ 


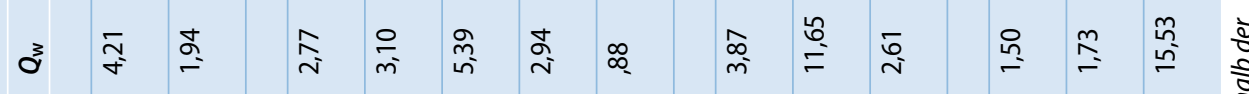

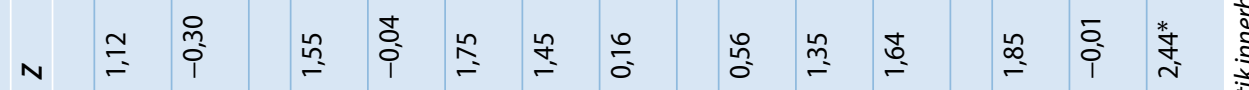

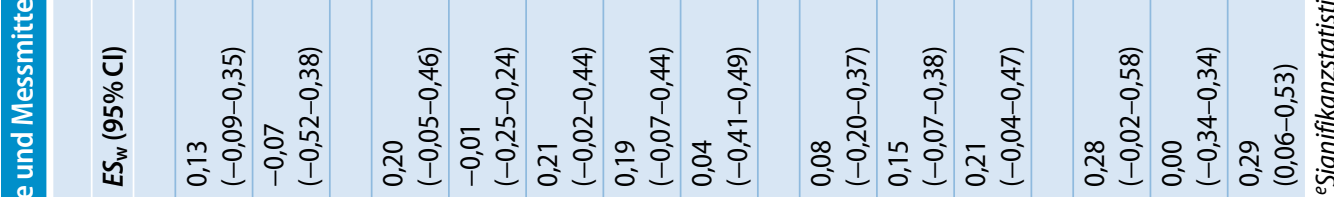

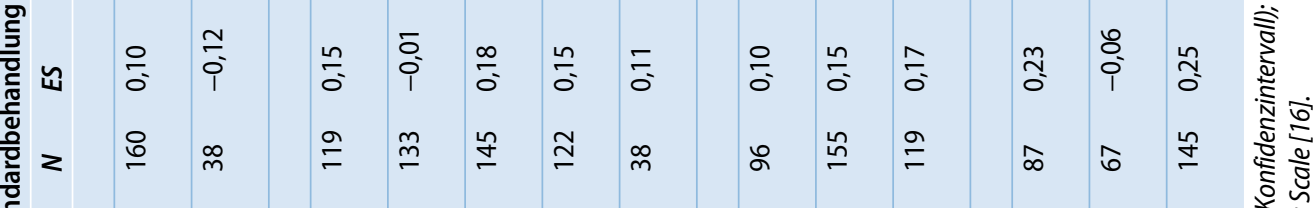

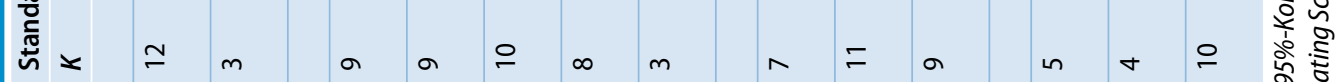

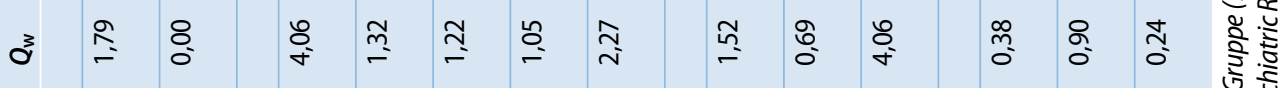

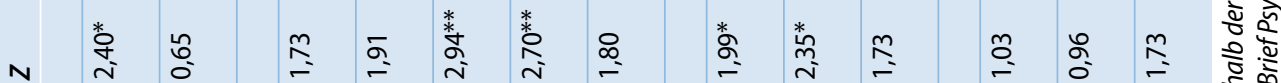

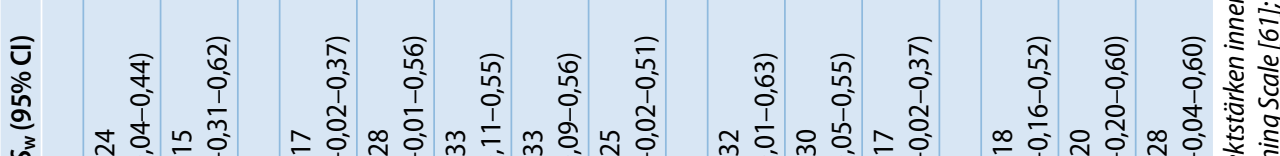

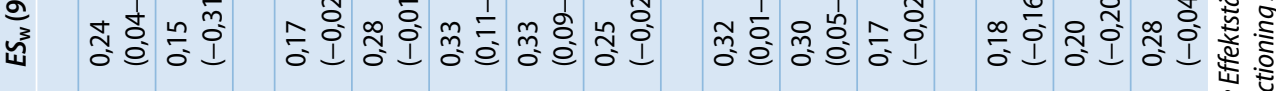
휸 z

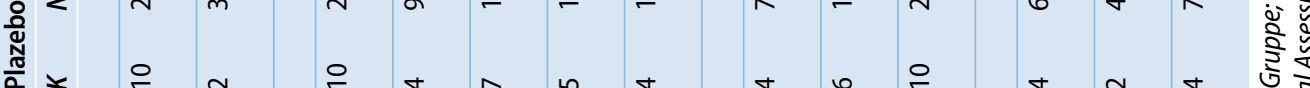

2 2 ก

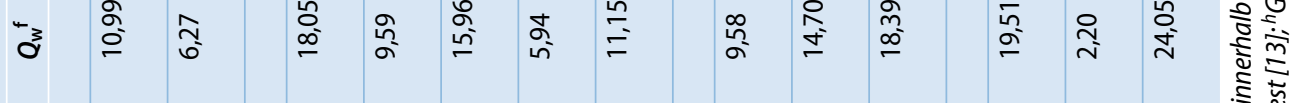

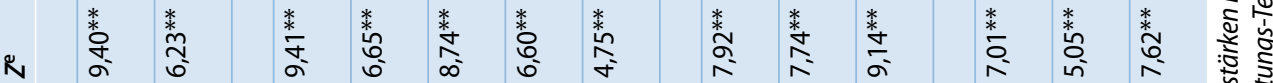
言

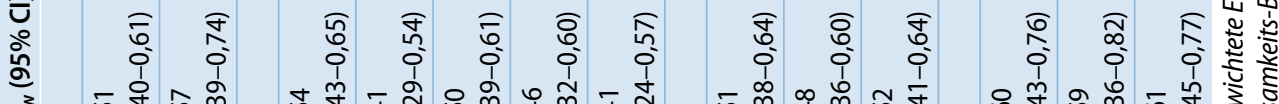

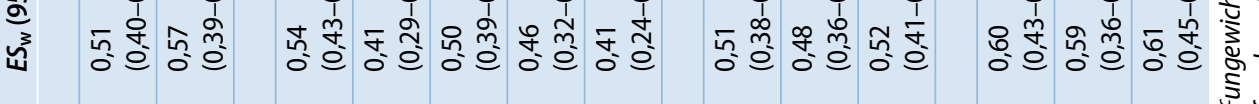

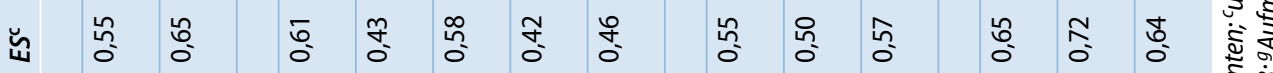

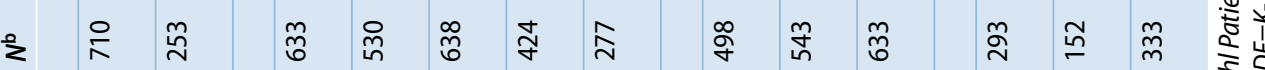

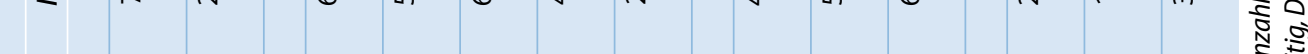

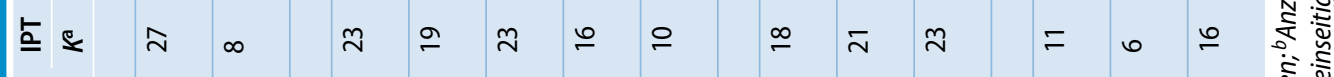

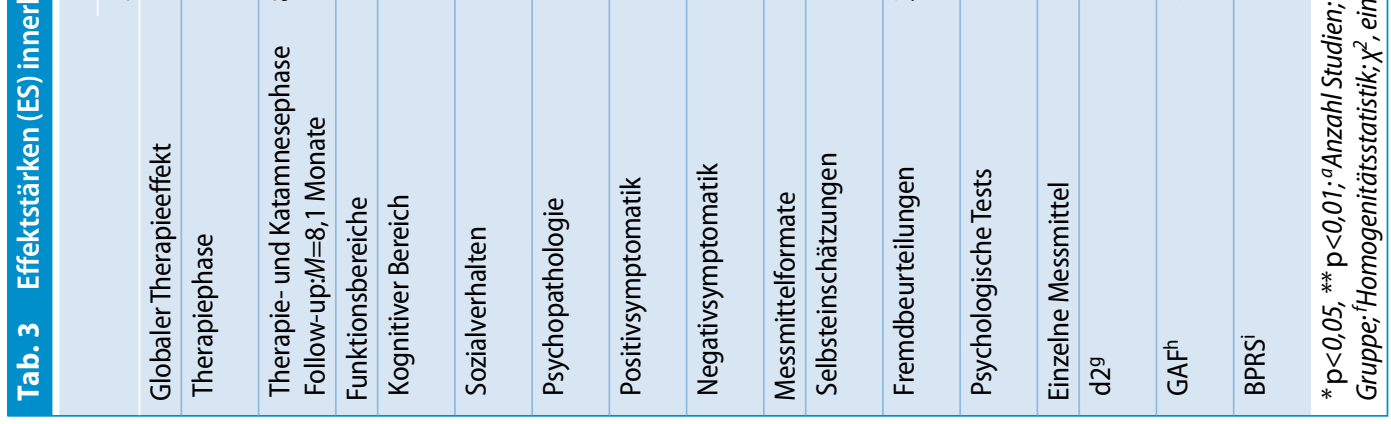


Tab. 4 Effektstärken (ES) innerhalb der IPT- Gruppen und der zusammengefassten Kontrollgruppen: globaler Therapieeffekt kontrolliert nach Zentren, Behandlungssetting und Krankheitsstatus

\begin{tabular}{|c|c|c|c|c|c|c|c|c|c|c|c|c|}
\hline & \multicolumn{6}{|l|}{ IPT } & \multicolumn{6}{|c|}{ Kontrollgruppen ${ }^{a}$} \\
\hline & $K^{b}$ & $N^{c}$ & $E S^{\mathrm{d}}$ & $E S_{\mathrm{w}}(95 \% \mathrm{Cl})^{\mathrm{e}}$ & $Z f$ & $Q_{w}{ }^{g}$ & $K$ & $N$ & ES & $E S_{\mathrm{w}}(95 \% \mathrm{Cl})$ & $Z$ & $Q_{w}$ \\
\hline \multicolumn{13}{|l|}{ Zentren } \\
\hline Universitäre Zentren: & 10 & 258 & 0,63 & $\begin{array}{l}0,56 \\
(0,38-0,73)\end{array}$ & $6,19^{* *}$ & 5,43 & 5 & 102 & 0,25 & $\begin{array}{l}0,31 \\
(0,01-0,61)\end{array}$ & $2,06^{*}$ & 1,63 \\
\hline Nichtuniversitäre Zentren & 13 & 288 & 0,51 & $\begin{array}{l}0,50 \\
(0,34-0,67)\end{array}$ & $5,92^{* *}$ & 2,90 & 13 & 230 & 0,09 & $\begin{array}{l}0,14 \\
(-0,05-0,32)\end{array}$ & 1,43 & 1,85 \\
\hline Multicenter $^{\mathrm{h}}$ & 4 & 90 & 0,47 & $\begin{array}{l}0,44 \\
(0,22-0,66)\end{array}$ & $3,96^{* *}$ & 2,01 & 2 & 30 & 0,06 & $\begin{array}{l}0,05 \\
(-0,46-0,56)\end{array}$ & 0,20 & 0,07 \\
\hline \multicolumn{13}{|l|}{ Behandlungssetting } \\
\hline Stationäre Patienten & 20 & 475 & 0,57 & $\begin{array}{l}0,53 \\
(0,40-0,66)\end{array}$ & $8,07^{* *}$ & 9,39 & 15 & 287 & 0,14 & $\begin{array}{l}0,20 \\
(0,03-0,37)\end{array}$ & $2,29^{*}$ & 3,81 \\
\hline Ambulante Patienten & 5 & 105 & 0,53 & $\begin{array}{l}0,49 \\
(0,22-0,77)\end{array}$ & $3,50^{* *}$ & 1,00 & 5 & 75 & 0,07 & $\begin{array}{l}0,08 \\
(-0,24-0,40)\end{array}$ & 0,50 & 0,55 \\
\hline \multicolumn{13}{|l|}{ Krankheitsstatus } \\
\hline $\begin{array}{l}\text { Symptomstabilisierte } \\
\text { Patienten }\end{array}$ & 20 & 446 & 0,57 & $\begin{array}{l}0,52 \\
(0,39-0,66)\end{array}$ & $7,67^{* *}$ & 9,39 & 16 & 264 & 0,10 & $\begin{array}{l}0,14 \\
(-0,04-0,32)\end{array}$ & 1,57 & 4,36 \\
\hline Postakute Patienten & 6 & 174 & 0,51 & $\begin{array}{l}0,50 \\
(0,29-0,72)\end{array}$ & $4,62^{* *}$ & 1,34 & 4 & 98 & 0,24 & $\begin{array}{l}0,25 \\
(-0,03-0,53)\end{array}$ & 1,73 & 0,04 \\
\hline
\end{tabular}

${ }^{*} \mathrm{p}<0,05,{ }^{* *} \mathrm{p}<0,01 ;{ }^{a}$ Plazebo-Attention-Bedingungen und Standardbehandlung zusammengefasst; ${ }^{b}$ Anzahl Studien; ${ }^{c}$ Anzahl Patienten; ${ }^{d}$ ungewichtete Effekt-

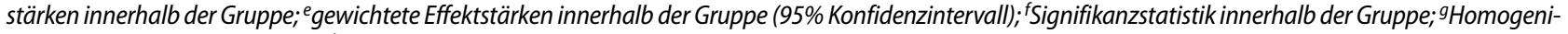
tätsstatistik: $X^{2}$ einseitig, $D F=K-2$; ${ }^{h}$ überwiegend nichtuniversitäre Zentren; ${ }^{i}$ stabiler Residualzustand.

$\left(Q_{B}=8,46 ; D F=1, p<0,01\right) . Z u$ Therapiebeginn weisen stationäre Patienten eine ausgeprägtere Symptomatik auf (BPRSGesamtscore [16]: $K=12 ; M \pm S D: 47,8 \pm 8,7$ ) als ambulante Patienten (BPRS-Gesamtscore: $K=4 ; 40,2 \pm 11,8)$.

\section{Krankheitsstatus}

Die IPT-Gruppe zeigt signifikante Effekte bei symptomstabilisierten und postakuten Patienten während der Therapiephase (• Tab. 4). Für die Kontrollgruppe können keine signifikanten Effekte nachgewiesen werden, doch erreichen postakute Patienten kleine $E S_{\mathrm{w}}$ und symptomstabilisierte Patienten keine Effekte. $\mathrm{Zu}$ Therapiebeginn weisen postakute IPTund Kontrollpatienten größere kognitive Defizite auf (D2 Standardwert [13]: $K=4$; $88,3 \pm 3,5)$ als symptomstabilisierte Patienten (D2 Standardwert: $K=5 ; 101,7 \pm 21,8$ ). Eine signifikante Überlegenheit des IPT gegenüber der Kontrollgruppe kann nur bei symptomstabilisierten Patienten nachgewiesen werden $\left(Q_{B}=11,17 ; D F=1\right.$, $p<0,01)$. Der Krankheitsstatus hat jedoch keinen Einfluss auf die Wirksamkeit des IPT $\left(Q_{B}=0,02, D F=1, n\right.$. s.). Für die Katamnesephase können aufgrund fehlender Datengrundlage nur ES von symptomstabilisierten IPT-Patienten berechnet werden. Diese können die während der Therapie erreichten Effekte auch in der Katamnese aufrecht erhalten (Followup =9,7 Monate; $K=6 ; E S=0,64 ; E S_{\mathrm{w}}=0,53$; CI: 0,29-0,77).

\section{Unterprogramme des IPT}

Das IPT wurde in den einzelnen Studien in unterschiedlicher Form eingesetzt. Bei 11 Studien kamen die UP „Kognitive Differenzierung“, „Soziale Wahrnehmung“ und „Verbale Kommunikation“ zum Einsatz (UP-Teil I); 5 Studien setzten dagegen nur die UP „Soziale Fertigkeiten“ und „Interpersonelles Problemlösen“ ein (UP-Teil II); 12 Studien verwendeten das gesamte IPT (IPT-Gesamt). Für alle drei IPT-Variationen können signifikante globale Therapieeffekte während der Therapiephase nachgewiesen werden (UPTeil I: $E S=0,58 ; E S_{\mathrm{w}}=0,58 ; \mathrm{CI}: 0,39-0,77$; UP-Teil II: $E S=0,54 ; E S_{\mathrm{w}}=0,52$; CI: $0,26-$ 0,78; IPT-Gesamt: $E S=0,51 ; E S_{\mathrm{w}}=0,46, \mathrm{CI}$ : o,32-0,61). Die $E S_{\mathrm{w}}$ der drei IPT-Variationen sind homogen $\left(Q_{B}=0,92, D F=2, n\right.$. s.). In den drei Funktionsbereichen erreichen Patienten unter UP-Teil I die höchsten gewichteten Effekte im kognitiven Bereich $\left(K=11 ; E S=0,72 ; E S_{\mathrm{w}}=0,71, \mathrm{CI}: 0,51-\right.$ $0,90)$ und die kleinsten im Sozialverhalten $\left(K=7 ; E S=0,38 ; E S_{\mathrm{w}}=0,37, \mathrm{CI}: 0,13-\right.$ $0,61)$ verglichen mit UP-Teil II und IPTGesamt. Im Intergruppenvergleich un- terscheiden sich die drei IPT-Variationen hinsichtlich der drei Funktionsbereiche jedoch nicht signifikant $\left(Q_{B}<4,59, D F=2\right.$, n. s.). Zum Vergleich der katamnestischen Ergebnisse werden aufgrund der geringen Zellengröße UP-Teil I und II zusammengefasst (UP-Teil I/II). Die Erhebungen nach einem Follow-up von durchschnittlich 8,3 Monaten nach Therapieende für UP-Teil I/II $(K=3)$ sowie 7,9 Monaten für IPT-Gesamt $(K=5)$ zeigen für beide Kategorien signifikante Verbesserungen gegenüber der Baseline. Jedoch ist $E S_{\mathrm{w}}$ der Therapie- und Katamnesephase für IPTGesamt ( $E S=0,74 ; E S_{\mathrm{w}}=0,60$; CI: o,39O,81) um $25 \%$ größer als für UP-Teil I/II $\left(E S=0,50 ; E S_{\mathrm{w}}=0,48, \mathrm{CI}: 0,13-0,82\right)$. Im direkten statistischen Vergleich unterscheiden sich die beiden IPT-Gruppen nicht $\left(Q_{B}=0,35 ; D F=1\right.$, n. s. $)$.

\section{Outcomeprädiktoren}

Von den Variablen der Patientencharakteristika, des Therapiesettings und der chronologischen Entwicklung der psychiatrischen Versorgung (Publikationsdatum, diagnostische Einschlusskriterien) hat einzig die Krankheitsdauer einen Einfluss auf den globalen Therapieeffekt ( $K=19$, Spearmans Rangkorrelation, zweiseitig: $r=-0,64 ; p<0,01)$. Zudem weist eine länge- 
Abb. 2 $>$ Gewichtete mittlere Effektstärke $\left(\mathrm{ES}_{\mathrm{w}}\right)$ der gesamten Stichprobe (GS; $\mathrm{K}=28$ ), von Studien in randomisiertem Design (RCT; $\mathrm{K}=14$ ) und von High-quality-Studien (HQS, K=7)

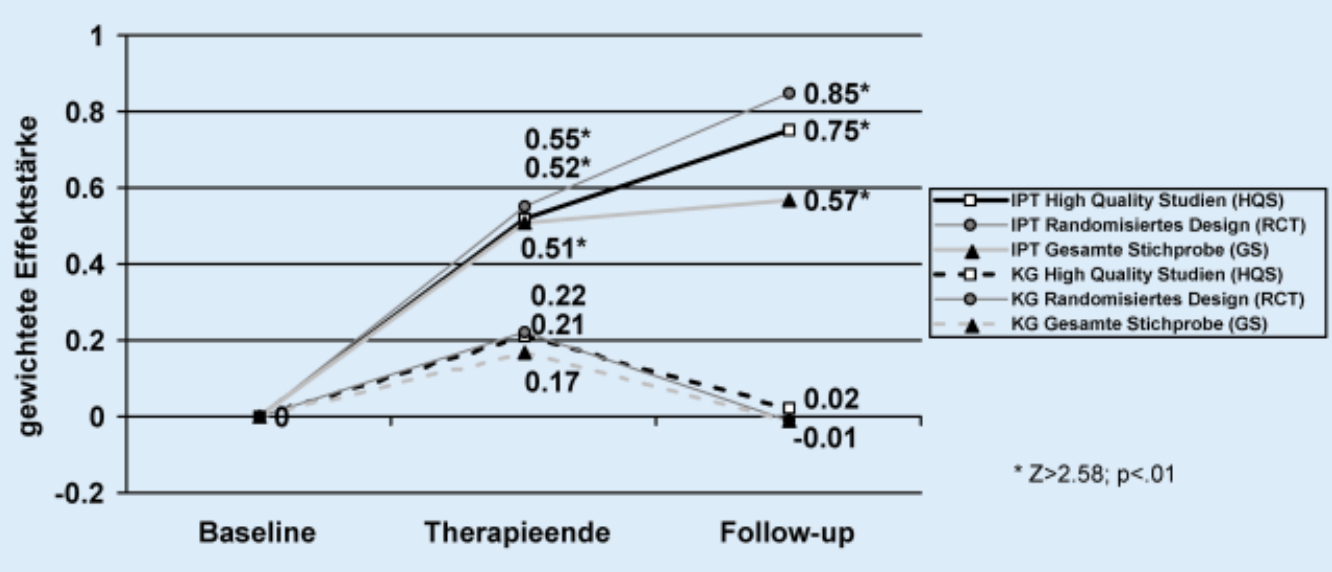

re Therapiedauer auf einen positiven Einfluss auf die Verbesserungen der sozialen Kompetenz hin $(K=19, r=0,47 ; p<0,05)$. Die einzige Studie mit einer Therapiefrequenz von nur einer Sitzung pro Woche [68] erzielt einen bedeutend kleineren mittleren Effekt $(E S=0,17)$.

Die drei Funktionsbereiche stehen während der Therapiephase in unterschiedlichem Zusammenhang. Während Verbesserungen im kognitiven Bereich signifikant mit solchen in der Psychopathologie $(K=19, r=0,52 ; p<0,05)$ und im Sozialverhalten $(K=15, r=0,51 ; p<0,05)$ korrelieren, können keine signifikanten $\mathrm{Zu}$ sammenhänge zwischen Psychopathologie und Sozialverhalten nachgewiesen werden ( $K=17, r=0,08$, n. s.). Eine Verringerung der Psychopathologie steht jedoch in moderatem Zusammenhang mit der Selbsteinschätzung des Sozialverhaltens $(K=7, r=0,71, \mathrm{n}$. s. $)$.

Des Weiteren korrelieren die mittleren Verbesserungen während einer katamnestischen Erhebung hochsignifikant mit jenen nach Therapieende $(K=8, r=0,91$; $p<0,01)$. Verlaufsprädiktoren für Followup-Erhebungen sind ebenfalls Veränderungen während der Therapie im kognitiven Bereich $(K=6, r=0,89 ; p<0,05)$ und in der Psychopathologie $(K=8, r=0,79$; $p<0,05)$. Verbesserungen in diesen beiden Bereichen stehen zudem in signifikant negativem Zusammenhang mit der Höhe der Drop-out-Rate $(K=11, r<-0,60$; $p<0,05)$.

\section{Effektstärken zwischen den Vergleichsgruppen}

Für die Berechnung der Effektstärken zwischen den Gruppen (d) wurden alle kont- rollierten Studien eingeschlossen $(K=21$; $N=900$ Patienten) und die beiden Kontrollbedingungen zusammengefasst. Patientencharakteristika und Setting unterscheiden sich nicht von jenen der Gesamtpopulation. Das IPT zeigt sowohl während der Therapie- als auch während der Katamnesephase gegenüber den Kontrollbedingungen zusätzliche globale Therapieeffekte (Therapiephase: $K=21 ; d=0,40$; $d_{\mathrm{w}}=0,36$, CI: 0,23-0,50; Therapie- und Katamnesephase: $K=6 ; d=0,52 ; d_{\mathrm{w}}=0,45$, CI: 0,19-0,71). Auch in den drei Funktionsbereichen kann eine Überlegenheit des IPT nachgewiesen werden (kognitiver Bereich: $K=18 ; d=0,46 ; d_{\mathrm{w}}=0,41, \mathrm{CI}$ : o,26-o,55; Sozialverhalten: $K=13 ; d=0,34$; $d_{\mathrm{w}}=0,31, \mathrm{CI}: 0,15-0,48$; Psychopathologie: $\left.K=16 ; d=0,31 ; d_{\mathrm{w}}=0,31, \mathrm{CI}: 0,16-0,46\right)$. Wird der globale Therapieeffekt $d$ in einen Korrelationskoeffizienten transformiert, entspricht dieser $r=0,20$. Dies bedeutet gemäß dem Binomial Effect Size Display (BESD) [6o], dass durchschnittlich $60 \%$ der IPT-Patienten und 40\% der Kontrollpatienten von der Behandlung erfolgreich profitieren. Zur Kontrolle des Publikationsbias wurde ermittelt, dass mindestens 108 IPT-Studien ohne Effekte $(Z=0, p=0,05)$ nötig wären, um die positiven Effekte von IPT gegenüber den Kontrollgruppen $(K=21 ; r=0,20, Z=0,89) \mathrm{zu}$ negieren [6o].

\section{Validieren der Ergebnisse}

Um methodische und designbedingte Störeinflüsse zu eliminieren, werden (a) nur Studien mit randomisierter Patientenzuteilung (Randomized Controlled Trials, RCT) und (b) nur Studien mit hoher wissenschaftlicher Qualität (High Quality
Studies, HQS) eingeschlossen. Vierzehn Studien mit insgesamt 604 Patienten erfüllen das RCT-Kriterien und 7 Studien mit insgesamt 362 Patienten die HQSKriterien. Weder RCT noch HQS unterscheiden sich von der gesamten Stichprobe (GS) hinsichtlich Patientencharakteristika (• Tab. 2) und Therapiesetting. Zwölf RCT und 6 HQS rekrutierten ausschließlich stationäre Patienten (je 85,7\%), 9 RCT $(64,3 \%)$ und 4 HQS (57,1\%) symptomstabilisierte Patienten. Sieben RCT (50\%) und 5 HQS $(71,4 \%)$ verglichen das IPT mit Plazebo-Attention-Bedingungen, 3 RCT (21,4\%) und eine HQS (14,3\%) mit Standardversorgung, 2 RCT und eine HQS (je 14,3\%) mit beiden Kontrollbedingungen. Zwei weitere RCT (14,3\%) verglichen IPT mit anderen Therapieansätzen. Sechs RCT und 3 HQS (je 42,9\%) wandten nur die IPT-Unterprogramme zum kognitiven Bereich (UP 1-3), 6 RCT (42,9\%) und 4 HQS $(57,1 \%)$ das gesamte IPT an. Zudem beinhalteten 4 HQS Blindratings $(57,1 \%)$ und 3 Studien $(42,9 \%)$ keine Fremdbeurteilungen.

Der globale Therapieeffekt der RCT und der restlichen Studien der GS (NICHT-RCT, $K=14$ ) sowie der der HQS und der NICHT-HQS $(K=21)$ unterscheiden sich weder in den IPT- noch in den Kontrollgruppen $\left(Q_{B}<0,75, D F=1, n\right.$. s.). Entsprechend sind auch die Ergebnisse von RCT, HQS und GS homogen (- Abb. 2).

Die Überlegenheit des IPT gegenüber den Kontrollbedingungen ist sowohl bei RCT $\left(Q_{\mathrm{B}}=6,24 ; \mathrm{DF}=1, p<0,05\right)$, HQS $\left(Q_{\mathrm{B}}=4,02 ; \mathrm{DF}=1, p<0,05\right)$ als auch bei $\mathrm{GS}$ $\left(Q_{\mathrm{B}}=12,66 ; \mathrm{DF}=1, p<0,01\right)$ signifikant. Die Effekte der RCT und der HQS über die Therapie- und Katamnesephase sind hö- 
her als jene der GS, jedoch vergleichbar mit jenen der Subpopulation stationärer Patienten der GS. Wiederum unterscheiden sich RCT und NICHT-RCT bzw. HQS und NICHT-HQS nicht $\left(Q_{B}<3,30\right.$; $\mathrm{DF}=1$, n. s.). Wie bei GS (• Tab. 3) zeigen auch die IPT-Patienten der RCT und HQS in allen drei Funktionsbereichen signifikante Ergebnisse: Kognitionen (RCT: $K=12 ; E S=0,63 ; E S_{\mathrm{w}}=0,54, \mathrm{CI}$ : o,37-0,71; HQS: $K=6 ; E S=0,52 ; E S_{\mathrm{w}}=0,48$, CI: $0,26-0,70$ ), Sozialverhalten (RCT: $K=8 ; E S=0,48 ; E S_{\mathrm{w}}=0,50, \mathrm{CI}: 0,30-0,71$ : HQS: $K=4 ; E S=0,55 ; E S_{\mathrm{w}}=0,62, \mathrm{CI}: 0,33-$ $0,92)$ und Psychopathologie (RCT: $K=12$; $E S=0,61 ; E S_{\mathrm{w}}=0,53$, CI: 0,37-0,70; HQS: $K=5 ; E S=0,50 ; E S_{\mathrm{w}}=0,49$, CI: $0,26-0,72$ ). Die Variable der Fremdbeurteilung ist aufgrund des Studienselektionskriteriums „Blindrating“ der HQS von besonderem Interesse. Verglichen mit der GS (- Tab. 3) besteht für die IPT-Gruppen kein Unterschied in der zusammengefassten Variable Fremdbeurteilung $(K=4$; $\left.E S=0,49, E S_{\mathrm{w}}=0,52, \mathrm{CI}: 0,35-0,60\right)$. Wird diese Variable in die beiden Funktionsbereiche Psychopathologie und Sozialverhalten unterteilt, so ist im Bereich der Psychopathologie ebenfalls kein Unterschied festzustellen (GS: $K=19 ; E S=0,51$; $E S_{\mathrm{w}}=0,45$, CI: $0,33-0,58$; HQS: $K=3$; $\left.E S=0,53 ; E S_{\mathrm{w}}=0,49 ; \mathrm{CI}: 0,17-0,81\right)$. Dagegen weisen die HQS in den fremdbeurteilten Variablen zum Sozialverhalten einen um $38 \%$ höheren Effekt auf als die GS (GS: $K=17 ; E S=0,43 ; E S_{\mathrm{w}}=0,45 ; \mathrm{CI}: 0,32$ 0,58; HQS: $K=4 ; E S=0,55 ; E S_{\mathrm{w}}=0,62 ; \mathrm{CI}$ : $0,33-0,92)$.

Auch die Berechnung von Effektstärken (d) zwischen IPT und den zusammengefassten Kontrollbedingungen der RCT und der HQS bestätigt die Ergebnisse der GS. Der gemittelte gewichtete Effekt während der Therapiephase der RCT $\left(K=11 ; d=0,41 ; d_{\mathrm{w}}=0,38, \mathrm{CI}: 0,19-\right.$ $0,57)$ und der HQS $\left(K=7 ; d=0,37 ; d_{\mathrm{w}}=0,35\right.$, CI: 0,14-0,57) sind nahezu identisch mit jenem der GS $\left(K=21 ; d=0,40 ; d_{\mathrm{w}}=0,36, \mathrm{CI}\right.$ : $0,23-0,50)$.

\section{Diskussion}

Die Wirksamkeit des IPT unter kontrollierten, randomisierten Bedingungen („efficacy“) konnte in mehreren Primärstudien nachgewiesen werden und ist in früheren Überblicksarbeiten dargestellt $[62,71]$. Der Fokus dieser Arbeit liegt auf der Wirksamkeit des IPT unter klinischen (Praxis-)Bedingungen („effectiveness“): Das IPT erzielt einen signifikant höheren globalen Therapieeffekt als unspezifische Gruppen und/oder Standardbehandlung, sowohl während der Therapie- als auch während der Katamnesephase. Dabei hat sich der unmittelbare Therapieerfolg nach Beendigung der Gruppen als Prädiktor für zusätzliche katamnestische Effekte erwiesen. Der Vergleich dieser Ergebnisse mit Befunden anderer Metaanalysen fällt jedoch z. T. widersprüchlich aus. Die Follow-up-Ergebnisse der IPT-Studien stimmen zwar mit der Mehrheit der heute vorliegenden Metaanalysen überein, die eine Aufrechterhaltung bzw. Erhöhung der Effekte in der Katamnesephase nachweisen [4, 23, 45, 52]. Auch die mittleren ES der Therapiephase der kontrollierten IPTStudien sind vergleichbar mit der Mehrheit relevanter Metaanalysen [4, 19, 35, 45, 76] sowie in 2 Primärstudien mit den Effekten anderer Therapieansätze [59, 73]. Über einen Zeitraum von 24 Jahren haben zudem der Zeitpunkt der jeweiligen Studie und das verwendete diagnostische Verfahren keinen Einfluss auf die gewichteten ES der Therapiephase. Die ES von IPT während der Therapiephase sind jedoch kleiner als die zum Teil großen ES von 3 Metaanalysen zu CBT und kognitiver Remediation [23, 39, 52].

Diese Ergebnisse und deren Interpretation unterliegen inhaltlichen und methodischen Bedingungen. Die unterschiedlich bewertete Effektivität („efficacy“) verschiedener kognitiv-behavioraler Interventionsansätze aufgrund von Studienselektion und limitierter Anzahl eingeschlossener Studien (statistische Validität) wird daher in der Literatur widersprüchlich diskutiert $[35,36,50]$. Es lassen sich folgende methodische Einflüsse zusammenfassen, anhand derer auch die Ergebnisse dieser Studie einzuordnen sind: Messmittelformate, operationalisierte Variablen, Funktionsbereiche und Versorgungsbedingungen.

\section{Messmittelformate}

Entgegen den Ergebnissen von Mojtabai et al. [45], aber in Übereinstimmung mit Dilk u. Bond [19] können keine Unterschiede in Fremd- und Selbstbeurteilungen nachgewiesen werden. Expertenbeurteilungen stellen in den eingeschlossenen Studien ein adäquates Abbild der Patientenselbsteinschätzungen dar. Auch Fremdbeurteilungen innerhalb nichtblinder Designs scheinen die Ergebnisse nicht zu verzerren.

\section{Operationalisierte Variablen}

In der Regel können bei kognitiv-behavioralen Ansätzen Effekte hinsichtlich des „primären Outcomes“, also in mit den Interventionen übereinstimmenden Bereichen, nachgewiesen werden [15]. Auf entsprechende empirische Hinweise verweisen einzelne IPT-Studien [20, 22]. Auch die höheren Effekte der kognitiven UP im kognitiven Bereich gegenüber anderen IPT-Variationen deuten auf die interne Validität dieser Ergebnisse hin. Dagegen liegen zum „sekundären Outcome“, also zur Generalisierung und zum Transfer der Ergebnisse widersprüchliche empirische Ergebnisse vor. Insbesondere Variablen zum generalisierten Sozialverhalten sind mit Störvariablen konfundiert (z. B. unspezifische Behandlungsaspekte) und methodologisch schwer zu operationalisieren [15]. In Übereinstimmung mit anderen Metaanalysen fallen diese beim IPT im Vergleich zu anderen Funktionsbereichen tendenziell kleiner aus $[45,51,52]$.

\section{Funktionsbereiche}

In sämtlichen Funktionsbereichen zeigt das IPT eine deutliche Überlegenheit gegenüber den Kontrollbedingungen. Im kognitiven Bereich weist das IPT die größten Effekte auf. Insbesondere die Effekte des ersten IPT-UP, das Strategielernen im Gruppenkontext vermittelt, stimmen mit metaanalytischen Ergebnissen zur kognitiven Remediation überein [35]. Im Bereich der Psychopathologie erreichen auch Patienten ohne psychotherapeutische Behandlung kleine Effekte, was auf die Wirkung von Psychopharmaka hinweist. Dagegen führt eine Medikation ohne zusätzliche Psychotherapie zu keiner positiven Veränderung der sozialen Kompetenz. Dies wird von empirischen Befunden zu sozialen Fertigkeits- und Problem- 
lösetrainings bestätigt $[28,34]$. Werden jedoch ausschließlich kognitive UP eingesetzt, fallen deren durchschnittliche Effekte im Sozialverhalten im Vergleich zu zusätzlicher oder alternativer Anwendung der UP zur sozialen Kompetenz tiefer aus. Zur Verbesserung der sozialen Kompetenz dürfte zudem eine längere Therapiedauer förderlich sein.

In Übereinstimmung mit integrativen Modellannahmen zur gegenseitigen Beeinflussung unterschiedlicher Funktionsebenen $[6,24]$ stehen die Katamneseergebnisse: Nur Patienten, die am gesamten IPT mit seinen Therapiekomponenten Neuro-, Sozialkognition und soziale Kompetenz teilnehmen, zeigen eine deutliche Effektzunahme während der Katamnese. Zusammen mit neueren Untersuchungen $[42,47,74,77]$ unterstützen auch diese Ergebnisse die Annahme der Generalisierung verbesserter Neurokognitionen und sozialer Fertigkeiten auf das allgemeine soziale Funktionsniveau.

\section{Versorgungsbedingungen}

Die differenzielle Wirksamkeit des IPT kann unabhängig von Patienten-, Settingund Rahmenbedingungen nachgewiesen werden. Die mittleren Effekte während der Therapiephase bei stationären und ambulanten Patienten stimmen mit Befunde anderer Metaanalysen überein [19, 45]. Bei der Interpretation der höheren Effekte stationärer Patienten in der Katamnese ist deren settingbedingte ausgeprägtere Symptomatik gegenüber ambulanten Patienten zu berücksichtigen. Auch symptomstabilisierte und postakute Patienten zeigen unter IPT signifikante Verbesserungen. Postakute Patienten weisen zu Therapiebeginn in der selektiven Aufmerksamkeit größere Defizite auf. Dieses Ergebnis wird durch die Zusammenfassung empirischer Ergebnisse zu neurokognitiven Defiziten von Wykes u. van der Gaag [77] unterstützt: Die selektive Aufmerksamkeit schizophren Erkrankter spiegelt episodenabhängige kognitive Defizite wider, die zwar auch prämorbid und in einer Remission nachweisbar sein können, in einer akuten psychotischen Episode jedoch stärker in den Vordergrund treten. Von anderen erhobenen Patientenvariablen konnte einzig die Krankheitsdauer als Erfolgsprädiktor bestimmt werden. Je länger Patienten erkrankt sind, desto geringer fallen deren Therapieeffekte aus. Der marginale Prädiktionswert der anderen Patientenvariablen wurde bereits bei Mojtabai et al [45] diskutiert. In Übereinstimmung mit anderen Studien $[32,35,45]$ besteht kein Einfluss des Therapiesettings auf die Therapieeffekte. Vor dem Hintergrund der Forderung nach Einbezug nichtuniversitärer Institutionen in die Psychotherapieforschung [69] können institutionsunabhängig signifikante IPT-Effekte nachgewiesen werden. In Übereinstimmung mit empirischen Ergebnissen [45] weisen Studien in universitären Zentren jedoch tendenziell höhere Effekte unter IPT- und Kontrollbedingungen auf.

\section{Fazit für die Praxis}

Die Ergebnisse der vorliegenden Metaanalyse unterstützen nicht nur die Evidenz des IPT als einen empirisch validierten Behandlungsansatz im Sinne der APARichtlinien, sondern haben auch weitreichende Konsequenzen für die klinische Praxis. Im Vergleich zu Ergebnissen von Einzelstudien, die aufgrund oft sehr spezifischer Eigenheiten des Settings und der teilnehmenden Patienten im klinischen Alltag kaum zu replizieren sind [60], berücksichtigt die Metaanalyse unter Einbezug unabhängiger Studien eine Vielzahl möglicher Effekteinflüsse. Die signifikant konsistenten Ergebnisse der vorliegenden Arbeit in unterschiedlichen klinischen Settings mit unterschiedlichen Merkmalen der partizipierenden Patienten gewährleistet dagegen mit erhöhter Wahrscheinlichkeit, dass im stationären, teilstationären oder ambulanten Rahmen unter einem breiten Spektrum psychiatrischer Versorgungsbedingungen vergleichbare Effekte erzielt werden können. Unter klinisch-praktischen, aber auch unter ökonomischen Gesichtspunkten können folgende Punkte für eine klinische Implementierung des IPT relevant sein:

In den 1980er Jahren wurde mit Therapiefrequenzen von bis zu 2-mal 5 Sitzungen pro Woche gearbeitet. In den letzten Jahren hat sich aus klinischen Erfahrungen abgeleitet und von empirischen Ergebnissen unterstützt ein Stan- dard von wöchentlich 2 IPT-Sitzungen durchgesetzt. Unter der Voraussetzung einer manualgestützten Durchführung möglichst homogener Therapiegruppen sind innerhalb eines multimodalen Behandlungsansatzes und bei entsprechender Indikationsstellung (vgl. Verhaltens- und Problemanalyse [55]) auch einzelne oder Kombinationen von IPT-Unterprogrammen sinnvoll, wirksam und kosteneffizient. Bei heterogenen Gruppen, unspezifischer Indikationsstellung und Patienten mit Defiziten auf mehreren Funktionsebenen bewirkt dagegen nur die Durchführung des gesamten IPT nachhaltige Effekte. Zudem dürfte die breite Anwendungsmöglichkeit des IPT bei Patienten mit unterschiedlichem Krankheits- und Rehabilitationsstatus im gesamten Spektrum psychiatrischer Versorgungseinrichtungen dazu beitragen, die Lücke zwischen gezielten kognitiv-behavioralen Interventionen und unspezifischen Rehabilitationsansätzen innerhalb der Standardversorgung schizophren Erkrankter zu schließen.

\section{Ausblick}

Die Ergebnisse unterliegen methodischen und inhaltlichen Einschränkungen. So ist die statistische Überprüfung der einbezogenen Studien von geringer Teststärke, was sich insbesondere bei den Ergebnissen zu ambulanten und postakuten Patienten gezeigt hat. Entsprechende Replikationsstudien sind daher wünschenswert. Aufgrund der Datengrundlage fehlen bisher fundierte Aussagen zur differenziellen Indikationsstellung unter Berücksichtigung individueller Rehabilitationsverläufe, über den Einfluss von Therapeutenvariablen und zur Rezidivprophylaxe. Zusätzlich müssen hinsichtlich einer wissenschaftlich fundierten Psychotherapie [14] auch Zusammenhänge differenzierter Funktionsbereiche während des Therapie- und Katamneseverlaufs, insbesondere in den Bereichen Neuro-, Sozialkognition und soziale Kompetenz, in weiteren kontrollierten Studien mit adäquater Stichprobengröße untersucht werden. 


\section{Korrespondierender Autor \\ Dr. V. Roder}

Universitätsklinik für Sozial- und Gemeindepsychiatrie, Universitäre Psychiatrische Dienste Bern

Bolligenstrasse 111, 3000 Bern 60

roder@spk.unibe.ch

Interessenkonflikt. Der korrespondierende Autor versichert, dass keine Verbindungen mit einer Firma, deren Produkt in dem Artikel genannt ist, oder einer Firma, die ein Konkurrenzprodukt vertreibt, bestehen.

\section{Literatur}

1. Becker T, Hulsmann S, Knudsen HC et al. (2002) Provision of services for people with schizophrenia in five European regions. Soc Psychiatry Psychiatr Epidemiol 37:465-474

2. Becker T, Vasquez-Barquero JL (2001) The european perspective of psychiatric reform. Acta Psychiatr Scand 104(Suppl 410):8-14

3. Bender W, Gerz L, John K et al. (1987) Kognitive Therapieprogramme bei Patienten mit schizophrener Residualsymptomatik. Untersuchungen über Wirksamkeit und klinische Erfahrungen. Neuropsychiatrie 2:212-217

4. Benton MK, Schroeder HE (1990) Socials skills training with schizophrenics: a meta-analytic evaluation. J Consult Clin Psychol 58:741-747

5. Blumenthal S, Bell V, Schüttler R et al. (1993) Ausprägung und Entwicklung von Basissymptomen bei schizophrenen Patienten nach einem kognitiven Therapieprogramm. Schizophrenie 8:20-28

6. Brenner HD (1986) Zur Bedeutung von Basisstörungen für Behandlung und Rehabilitation. In: Böker W, Brenner HD (Hrsg) Bewältigung der Schizophrenie. Huber, Bern, S 142-157

7. Brenner HD, Hodel B, Kube G et al. (1987) Kognitive Therapie bei Schizophrenen: Problemanalyse und empirische Ergebnisse. Nervenarzt 58:72-83

8. Brenner HD, Junghan U, Pfammatter M (2000) Gemeindeintegrierte Akutversorgung. Möglichkeiten und Grenzen. Nervenarzt 71:691-699

9. Brenner HD, Roder V, Hodel B et al. (1994) Integrated psychological therapy for schizophrenic patients. Hogrefe \& Huber, Seattle

10. Brenner HD, Seeger G, Stramke WG (1980) Evaluation eines spezifischen Therapieprogramms zum Training kognitiver und kommunikativer Fähigkeiten in der Rehabilitation chronisch schizophrener Patienten in einem naturalistischen Feldexperiment. In: Hautzinger D, Schulz W (Hrsg) Klinische Psychologie und Psychotherapie, Bd. 4. GWG/DGVT Köln, S 31-46

11. Brenner HD, Stramke WG, Brauchli B (1982) Integriertes psychologisches Therapieprogramm bei chronisch schizophrenen Patienten: Untersuchungen zur Differentialindikation. In: Helmchen $\mathrm{H}$, Linden M, Rueger U (Hrsg) Psychotherapie in der Psychiatrie. Springer, Berlin Heidelberg New York, S 77-85

12. Briand C, Lesage A, Lalonde P et al. (2003) The IPT for patients with schizophrenia: evidence of effectiveness during program implementation in various sites in Quebec, Canada. Schizophr Res 60(Suppl 1):320

13. Brickenkamp R (1975) Aufmerksamkeits-Belastungs-Test (Test d2). Hogrefe, Göttingen
14. Buchkremer G, Klingberg S (2001) Was ist wissenschaftlich fundierte Psychotherapie? Nervenarzt 72:20-30

15. Bustillo JR, Lauriello J, Horan WP et al. (2001) The psychosocial treatment of schizophrenia: an update. Am J Psychiatry 158:163-175

16. CIPS Collegium Internationale Psychiatriae Scalarum (1981) Internationale Skalen für Psychiatrie. Beltz, Weinheim

17. Cohen J (1988) Statistical power analyses for the behavioral sciences. Erlbaum, Hillsdale

18. Davis JM, Chen N, Glick ID (2003) A meta-analysis of the effecacy of second-generation antipsychotics. Arch Gen Psychiatry 60:553-564

19. Dilk MD, Bond GR (1996) Meta-analytic evaluation of skills training research for individuals with severe mental illness. J Consult Clin Psychol 64:1337-1346

20. Funke B, Reinecker H, Commichau A (1989) Grenzen kognitiver Therapiemethoden bei schizophrenen Langzeitpatienten. Nervenarzt 60:750756

21. Gaag van der M (1992) The results of cognitive training in schizophrenic patients. Eburon, Delft

22. Garcia S, Fuentes I, Ruiz JC et al. (2003) Application of the IPT in a Spanish sample of the "Social Perception Subprogramme". Int J Psychol Psychol Therapy 3:299-310

23. Gould RA, Mueser KT, Bolton E et al. (2001) Cognitive therapy for psychosis in schizophrenia: an effect size analysis. Schizophr Res 48:335-342

24. Green MF, Nuechterlein KH (1999) Should schizophrenia be treated as a neurocognitive disorder? Schizophr Bull 25:309-318

25. Harvey PD, Keefe RSE (2001) Studies of cognitive change in patients with schizophrenia following novel antipsychotic treatment. Am J Psychiatry 158:176-184

26. Hedges LV (1994) Fixed effects models. In: Cooper $\mathrm{H}$, Hedges LV (eds) The handbook of research synthesis. Sage, New York, pp 285-300

27. Heim M, Wolf S, Göthe U et al. (1989) Kognitives Training bei schizophrenen Erkrankungen. Psychiatr Neurol Med Psychol 41:367-375

28. Heinssen RK, Liberman RP, Kopelowicz A (2000) Psychosocial skills training for schizophrenia: lessons from the laboratory. Schizophr Bull 26:21-46

29. Hermanutz M, Gestrich J (1987) Kognitives Training mit Schizophrenen. Nervenarzt 58:91-96

30. Hodel B (1994) Reaktionsdefizite und ihre Wirkungen auf den Therapieerfolg bei schizophren Erkrankten. Schizophrenie 9:31-38

31. Hodel B, Brenner HD (1996) Ein Trainingsprogramm zur Bewältigung von maladaptiven Emotionen bei schizophren Erkrankten. Erste Ergebnisse und Erfahrungen. Nervenarzt 67:564-571

32. Hogarty GE, Flesher S, Ulrich R et al. (2005) Cognitive enhancement therapy for schizophrenia: effects of a two-year randomized trial on cognition and behavior. Arch Gen Psychiatry (in press)

33. Hubmann W, John K, Mohr F et al. (1991) Soziales Verhaltenstraining nit chronisch schizophrenen Patienten. In: Schüttler R (Hrsg) Theorie und Praxis kognitiver Therapieverfahren bei schizophrenen Patienten. Zuckschwerdt, München, S 118-128

34. Huxley NA, Rendall M, Sederer L (2000) Psychosocial treatment in schizophrenia. A review of the past 20 years. J Nerv Ment Dis 188:187-201

35. Krabbendam L, Aleman A (2003) Cognitive reabilitation in schizophrenia: a quantitative analysis of controlled studies. Psychopharmacology 169:376382
36. Krabbendam L, Aleman A (2003) Commentary: psychological treatment in schizophrenia: Il. Metaanalyses of randomized controlled trials of social skills training and cognitive remediation (Pilling et al. 2002, Psychol Med 32:783-791). Psychol Med $33: 756$

37. Kraemer S, Sulz KHD, Schmid R et al. (1987) Kognitive Therapie bei standardversorgten schizophrenen Patienten. Nervenarzt 58:84-90

38. Kraemer S, Zinner HJ, Riehl T et al. (1990) Kognitive Therapie und verhaltenstraining zur Förderung sozialer kompetenz für chronisch schizophrene $\mathrm{Pa}$ tienten. In: Kühne GE, Brenner HD, Huber G (Hrsg) Kognitive Therapie bei Schizophrenen. Fischer, Jena, S 73-82

39. Kurtz MM, Moberg PJ, Gur RC et al. (2001) Approaches to cognitive remediation of neuropsychological deficits in schizophrenia: a review and meta-analysis. Neuropsychol Rev 11:197-210

40. Lehman AF, Steinwachs DM (2003) Evidence-based psychological treatment practices in schizophrenia: lessons from the Patient Outcomes Research Team (PORT) project. J Am Acad Psychoanal Dyn Psychiatry 31:141-154

41. Liberman RP, Massel HK, Mosk MD et al. (1985) Social skills training for chronic mental patients. Hosp Community Psychiatry 36:396-403

42. Liddle PF (2000) Cognitive impairment in schizophrenia: its impact on social functioning. Acta Psychiatr Scand 101:11-16

43. Meichenbaum DW (1977) Methoden der Selbstinstruktion. In: Kanfer F, Goldstein AP (Hrsg) Möglichkeiten der Verhaltensänderung. Urban \& Schwarzenberg, München

44. Möller HJ (2000) Aktuelle Bewertung neuer/atypischer Neuroleptika. Nervenarzt 71:329-344

45. Mojtabai R, Nicholson RA, Carpenter BN (1998) Role of psychosocial treatment in management of schizophrenia: a meta-analytic review of controlled outcome studies. Schizophr Bull 24:569-587

46. Olbrich R, Mussgay L (1990) Reduction of schizophrenic deficits by cognitive training. An evaluative study. Eur Arch Psychiatry Clin Neurosci 239:366-369

47. Penades R, Boget T, Catalan R et al. (2003) Cognitive mechanisms, psychosocial functioning, and neurocognitive rehabilitation in schizophrenia. Schizophr Res 63:219-227

48. Peter K, Glaser A, Kühne GE (1989) Erste Erfahrungen mit der kognitiven Therapie Schizophrener. Psychiatr Neurol Med Psychol 41:485-491

49. Peter K, Kühne GE, Schlichter A et al. (1992) Ergebnisse der kognitiven Therapie und der Verlauf schizophrener Psychosen im ersten bis zweiten Jahr nach der Entlassung. Zur Problematik und Langzeitwirkung kognitiver Therapie. In: Brenner HD, Böker W (Hrsg) Verlaufsprozesse schizophrener Erkrankungen. Huber, Bern, S 350-361

50. Pilling S, Bebbington P, Kuipers E et al. (2002) Psychological treatment in schizophrenia: II. Metaanalyses of randomized controlled trials of social skills training and cognitive remediation. Psychol Med 32:783-791

51. Pilling S, Bebbington P, Kuipers E et al. (2002) Psychological treatment in schizophrenia: I. Metaanalyses of family intervention and cognitive behaviour therapy. Psychol Med 32:763-782

52. Rector NA, Beck AT (2001) Cognitive behavioral therapy for schizophrenia: an empirical review. J Nerv Ment Dis 189:278-287

53. Roder V (1990) Evaluation einer kognitiven Schizophrenietherapie. In Kühne GE, Brenner HD, Huber $\mathrm{G}(\mathrm{Hrsg})$ Kognitive Therapie bei Schizophrenen. Fischer, Jena, S 27-39 
54. Roder V, Brenner HD, Müller D et al.(2001) Effekte neuer kognitiv-behavioraler Therapieprogramme zur Verbesserung spezifischer sozialer Fertigkeiten bei schizophren Erkrankten: eine kontrollierte Studie. Nervenarzt 72:709-716

55. Roder V, Brenner HD, Kienzle N (2002) Integriertes Psychologisches Therapieprogramm für schizophren Erkrankte (IPT), 5. völlig neu bearbeitete Aufl. Beltz, Weinheim

56. Roder V, Brenner HD, Kienzle N et al. (1988) Integriertes Psychologisches Therapieprogramm (IPT) für schizophrene Patienten. Psychologie Verlags Union, München

57. Roder V, Studer K, Brenner HD (1987) Erfahrungen mit einem integrierten psychologischen Therapieprogramm zum Training kommunikativer und kognitiver Fähigkeiten in der Rehabilitation schwer chronisch schizophrener Patienten. Schweiz Arch Neurol Psychiatr 138:31-44

58. Roder V, Zorn P, Andres K et al. (2002) Praxishandbuch zur verhaltenstherapeutischen Behandlung schizophren Erkrankter. Huber, Bern

59. Roder V, Zorn P, Brenner HD (2000) Kognitiv-behaviorale Programme für schizophren Erkrankte zum Aufbau sozialer Kompetenz im Wohn-, Arbeitsund Freizeitbereich: Überblick und empirische Ergebnisse. Verhaltenstherapie und psychosoziale Praxis 32:195-211

60. Rosenthal R (1994) Meta-analytic procedures for social research. Sage Publications, Newbury Park

61. Sass H, Wittchen HU, Zaudig M (1996) Diagnostisches und statistisches Manual psychischer Störungen. DSM-IV. Übersetzt nach der vierten Auflage des Diagnostic and Statistical Manual of Mental Disorders der American Psychiatric Association. Hogrefe, Göttingen

62. Schüttler R, Bell V, Blumenthal S et al. (1990) Haben „kognitive“ Therapieprogramme messbaren Einfluss auf Basissymptome bei Schizophreneien? In: Huber G (Hrsg) Indiopathische Psychosen. Psychopathologie, Neurobiologie, Therapie. Schattauer, Stuttgart, S 219-240

63. Shadish WR, Haddock CK (1994) Combining estimates of effect size. In: Cooper H, Hedges LV (eds) The handbook of research synthesis. Sage, New York, pp 261-281

64. Smith ML, Glass GV (1977) Meta-analysis of psychotherapy outcome studies. Am Psychol 32:752760

65. Spaulding WD, Reed D, Sullivan M et al. (1999) Effects of cognitive treatment in psychiatric rehabilitation. Schizophr Bull 25:657-676

66. Spitzer R, Endicott J, Robins E (1977) Research Diagnostic Criteria (RDC) for a selected group of functional disorders, 3rd edn. Biometric Research, State Psychiatric Institute, New York

67. Stramke WG, Hodel B (1983) Untersuchungen zur Wirksamkeit psychologischer Therapieprogramme in der Rehabilitation chronisch schizophrener Patienten. In: Brenner HD, Rey ER, Stramke WG (Hrsg) Empirische Schizophrenieforschung. Huber, Bern, S 216-234

68. Takai A, Uematsu M, Kadama Y et al. (1993) Kognitives Therapieprogramm bei chronisch schizophrenen Japanern. Eine kontrollierte Therapiestudie über die Auswirkungen auf Symptomatik und Bewältigungsmechanismen. Schizophrenie 8:2934

69. Teusch L, Gastpar M (2000) Psychotherapieforschung in der Psychiatrie. Nervenarzt 71:213-217

70. Theilemann S (1993) Beeinflussung kognitiver Störungen bei schizophrenen und schizoaffektiven Psychosen mit Hilfe kognitiver Therapie im Vergleich zur Soziotherapie. Nervenarzt 64:587-593
71. Theilemann S, Peter K (1994) Zur Evaluation kognitiver Therapie bei schizophren Erkrankten: Überblicksarbeit. Z Klin Psychol 23:20-33

72. Vallina-Fernandez $\mathrm{O}$, Lemos-Giraldez $\mathrm{S}$, Roder V et al. (2001) Controlled study of an integrated psychological intervention in schizophrenia. Eur J Psychiatry 15:167-179

73. Vauth R, Joe A, Seitz M et al. (2001) Differenzielle Kurz- und Langzeitwirkung eines „Trainings Emotionaler Intelligenz" und des "Integrierten Psychologischen Therapieprogramms" für schizophrene $\mathrm{Pa}$ tienten. Fortschr Neurol Psychiatr 69:518-525

74. Velligan DI, Bow-Thomas C, Mahurin R et al. (2000) Do specific neurocognitive deficits predict specific domains of community function in schizophrenia? J Nerv Ment Dis 188:518-524

75. Vita A, Cocchi A, Contini A et al. (2002) Applicazione multicentrica del metodo riabilitativo strutturato IPT (Terapia Psicologica Integrata) per pazienti schizofrenici. Psichiatr Oggi 15:11-18

76. Wunderlich U, Wiedemann G, Buchkremer G (1996) Sind psychosoziale Interventionen bei schizophrenen Patienten wirksam? Eine Metaanalyse. Verhaltenstherapie 6:4-13

77. Wykes T, Gaag van der M (2001) Is it time to develop a new cognitive therapy for psychosis-Cognitive Remediation Therapy (CRT)? Clin Psychol Rev 21:1227-1256

78. Wykes T, Reeder C, Corner J et al. (1999) The effects of neurocognitive remediation on executive processing in patients with schizophrenia. Schizophr Bull 25:291-307
Haut und Psyche

Erworbene und angeborene Hautkrankheiten besitzen meist ein multifaktorielles Krankheitsbild. Neben den körperlichen Aspekten spielen die psychischen und sozialen Auswirkungen solcher Erkrankungen eine nicht zu unterschätzende Rolle. Da Beeinträchtigungen der Hautfunktion unterschiedliche Folgen auf Personen und deren Umfeld haben können, sollten bei einer Behandlung auch verhaltenstherapeutische Maßnahmen mit in Betracht gezogen werden.

In Ausgabe 11/2005 der Springer-Fachzeit-

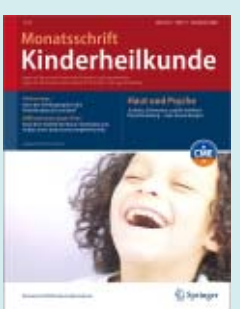
schrift „Monatsschrift Kinderheilkunde" finden Sie einen Überblick über eine Reihe von Techniken und Anregungen für das breite Feld einer Psychosomatik der

Haut. Informieren Sie sich in dieser Ausgabe u. a. über:

- Verhaltenstherapie bei Neurodermitis

- Xeroderma pigmentosum. Eine zerstörerische Hauterkrankung

- Verbrennungen bei Kindern. Intensivbehandlung und psychische Folgen

- Verbrennungen im Kindes- und Jugendalter. Psychische und soziale Folgen

- Hautbefunde bei Kindesmisshandlung. Machen blaue Flecke krank?

- Selbstverletzendes Verhalten

Hier können Sie diese Ausgabe für 27 Euro bestellen: Springer-Verlag

Kundeservice Zeitschriften

Haberstr. 7

69126 Heidelberg

Tel.: +49 6221-345-4303

Fax: +49 6221-345-4229

E-Mail: subscriptions@springer.com

MonatsschriftKinderheilkunde.de 